\title{
STATISTICAL CLUSTER POINTS OF SUBSEQUENCES OF DOUBLE SEQUENCES
}

\author{
HARRY I. MILLER
}

Dedicated to the memory of Professor Mahmut Bajraktarević

\begin{abstract}
Tha main result of this paper is the double sequence analogue of a theorem about cluster points of single sequences that has recently submitted for publication [8]. Namely, we show here that if $x$ is a double sequence then $\Gamma_{x}$ (the set of statistical cluster points of $x$ ) equals $\Gamma_{y}$ (the set of statistical cluster points of $y$ ) for "almost all" subsequences $y$ of $x$.
\end{abstract}

\section{INTRODUCTION}

If $t \in(0,1]$, then $t$ has a unique binary expansion $t=\sum_{n=1}^{\infty} b_{n} / 2^{n}, b_{n} \in$ 0,1 , with infinitely many of the $b_{n}{ }^{\prime} s$ being 1 ; so that for $t=1 / 2$ we use the representation

$$
\frac{1}{2}=\sum_{n=1}^{\infty} \frac{b_{n}}{2^{n}}
$$

with $b_{1}=0$ and all the other $b_{n}{ }^{\prime} s$ equal to 1 . Next, if $x=\left(x_{n}\right)_{n=1}^{\infty}$ is a sequence of reals, $x(t)$ denotes the subsequence of $x$ obtained using the terms (and only those terms) of $x$ for which $b_{n}=1$. For example if

$$
t=\frac{0}{2}+\frac{1}{2^{2}}+\frac{1}{2^{3}}+\frac{0}{2^{4}}+\frac{1}{2^{5}}+\ldots,
$$

then $x(t)$ is the subsequence $\left\{x_{2}, x_{3}, x_{5}, \ldots\right\}$ of $x$. Statistical convergence of a sequence $x=\left(x_{n}\right)$ is defined as follows:

The statistical limit of $x$ is $L$ (see [1], [5]), if for every $\varepsilon>0$,

$$
\lim _{n \rightarrow \infty} \frac{1}{n}\left|\left\{k \leq n|| x_{k}-L \mid \geq \varepsilon\right\}\right|=0
$$

where here $|A|$ denotes the number of elements in the finite set $A$.

2010 Mathematics Subject Classification. 40D25, 40G99, $28 \mathrm{~A} 12$.

Key words and phrases. Subsequences, double sequences, statistical cluster points.

Copyright (c) 2016 by ANUBIH. 
Statistical convergence and its connection to subsequences, was studied in $[5]$.

In the paper [2], "Statistical Limit Points", subsequences of $x$, for single sequences $x$, are considered.

In this paper, three concepts are examined.

$L_{x}$, the set of limit points of $x$;

$\Lambda_{x}$, the set of statistical limit points of $x$ and

$\Gamma_{x}$, the set of statistical cluster points of $x$.

The number $C$ is a statistical cluster point of $x$ provided that for every $\varepsilon>0$, the set

$$
\left\{k \in \mathbb{N}|| x_{k}-C \mid<\varepsilon\right\}
$$

does not have density zero, or is not the case that

$$
\lim _{n \rightarrow \infty} \frac{1}{n}\left|\left\{k \leq n|| x_{k}-C \mid<\varepsilon\right\}\right|=0,
$$

i.e. this limit is positive or does not exist. As Fridy mentioned, if $x$ is bounded, then $\Gamma_{x} \neq \emptyset$ and $\Gamma_{x}$ is always a closed set.

In [8] the following theorem is proved.

Theorem. If $x$ is a bounded sequence then $\Gamma_{x}=\Gamma_{x(t)}$ for almost all (in the sense of Lebesgue measure) $t \in(0,1]$.

\section{Preliminaries}

We need first to define what we mean by subsequences of a double sequence and what we mean by a statistical cluster point of a subsequences of a double sequence.

There are different ways to define statistical cluster points of a double sequence $x=\left(x_{i j}\right)$, subsequences $y$ of $x$ and statistical cluster points of $y$, and what we mean by "almost all" subsequences $y$ of $x$.

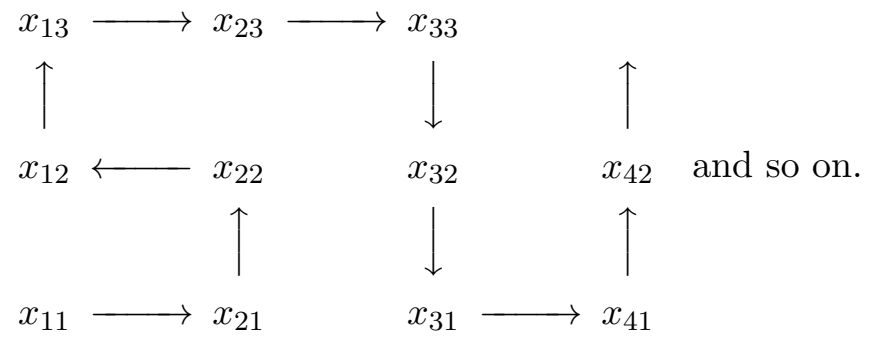

Using the diagram, we can convert a double sequence $x=\left(x_{i j}\right)$ into a single sequence $Z(x)=\left(Z_{n}\right)$. 
Definition 1. We define $z(x)=\left(z_{n}\right)$ to be the single sequence using our diagram by

$$
Z=\left(Z_{n}\right)=\left\{x_{11}, x_{21}, x_{22}, x_{12}, x_{13}, x_{23}, x_{33}, x_{32}, x_{31}, x_{41}, x_{42}, \ldots\right\} .
$$

Definition 2. Let $U=\left\{u=\left(u_{i j}\right) \mid u_{i j} \in\{0,1\}\right.$ for all $\left.i, j\right\}$.

Definition 3. Given a double sequence $x=\left(x_{i j}\right)$ and $u=\left(u_{i j}\right)$ in $U$ we define the subsequence $x(u)$ of $x$, determined by as follows:

$$
(x(u))_{i j}=\left\{\begin{array}{ll}
x_{i j} & \text { if } u_{i j}=1 \\
(*) & \text { if } u_{i j}=0
\end{array} .\right.
$$

Notice $x(u)$ is a double sequence. Again, using our diagram, we can convert $x(u)$ into a single sequence $Z(x(u))$, ignoring terms where $u_{i j}=0$.

Example 1. If $u_{11}=1, u_{21}=1, u_{22}=0, u_{12}=1, u_{13}=1, u_{23}=0, u_{33}=$ $0, u_{32}=1, u_{31}=0, u_{41}=1 \ldots$ then

$$
Z(x(u))=\left\{x_{11}, x_{21}, x_{12}, x_{13}, x_{32}, x_{41}, \ldots\right\} .
$$

Definition 4. $L$ is defined to be a statistical cluster point of the double sequence $x$, if $L \in \Gamma_{Z}$, where $Z=Z(x)$ is defined in Definition 1 and $\Gamma_{Z}$ is as defined in the introduction. Furthermore $\Gamma_{Z}$ is defined to be all such numbers $Z$.

Definition 5. $L$ is defined to be a statistical cluster point of the subsequence $x(u)$ if $L \in \Gamma_{Z(x(u))}$, where $Z(x(u))$ is the single sequence obtained, using the diagram, from the subsequence $x(u)$. All such $L$ will be defined to be $\Gamma_{x(u)}$.

By the Kolmogorov Consistency Theoren [3, pg.92] there exist a unique complete probability measure $P$, on a $\sigma$-algebra of subsets $\mathcal{M}$ of $U$ such that for each finite collection of pairwise distinct elements $\left(i_{1} j_{1}\right),\left(i_{2} j_{2}\right), \ldots$ $\left(i_{n} j_{n}\right) \in \mathbb{N} \times \mathbb{N}$ and each of the choices $t_{1}, t_{2}, \ldots, t_{n} \in\{0,1\}$ we have

$$
P\left(\left\{u=\left(u_{i j}\right) \mid u_{i_{1} j_{1}}=t_{1}, u_{i_{2} j_{2}}=t_{2}, \ldots, u_{i_{n} j_{n}}=t_{n}\right\}\right)=\frac{1}{2^{n}} .
$$

\section{Result}

We are now prepared to prove the theorem mentioned in our abstract.

Theorem 1. For any double sequence $x$

$$
P\left(\left\{u \in U: \Gamma_{x}=\Gamma_{x(u)}\right\}\right)=1 .
$$


Proof. We prove this result by showing two inclusion results. As mentioned before, Fridy has shown $\Gamma_{x}$ is always closed for single sequences. Minor modifications show $\Gamma_{x}$ and $\Gamma_{x(u)}$ are both closed for double sequence $x$ and $u \in U$. We first prove:

$$
P\left(\left\{u \in U: \Gamma_{x} \subseteq \Gamma_{x(u)}\right\}\right)=1 .
$$

Since $\Gamma_{x}$ is closed, either $\Gamma_{x}$ is finite or

$$
\Gamma_{x} \text { is closure of a set }\left\{L_{n}: n \in \mathbb{N}\right\} .
$$

We only offer the proof in the latter case, since the proof in the finite case is easier. Suppose we are able to show

$$
L_{n} \in \Gamma_{x(u)} \text { for all } u \in B_{n},
$$

where $P\left(B_{n}\right)=1$ and all $u \in B$, where $B=\bigcap_{n=1}^{\infty} B_{n}$ and $P(B)=1$. Hence $\left\{L_{1}, L_{2}, \ldots\right\} \subseteq \Gamma_{x(u)}$ for all $u \in B$. Taking closures and using $\Gamma_{x(u)}$ is always closed, we obtain the closure of $\left\{L_{1}, L_{2}, \ldots\right\} \subseteq \Gamma_{x(u)}$ for all $u \in B$ and therefore

$$
\Gamma_{x} \subseteq \Gamma_{x(u)} \text { for all } u \in B .
$$

So, in order to prove (1) it is sufficient to prove $L_{n} \in \Gamma_{x(u)}$ for all $u \in B_{n}$, or that (i) holds. To see this observe that for every $\varepsilon>0$

$$
\left\{i|i \in \mathbb{N},| Z_{i}-L_{n} \mid<\varepsilon\right\}
$$

is non-thin, where $Z=\left(Z_{i}\right)=Z(x)$. see Definition 4 . For each $l \in \mathbb{N}$, set $\varepsilon=1 / l$ we have

$$
\left\{i|i \in \mathbb{N},| Z_{i}-L_{n} \mid<\varepsilon\right\}
$$

is non-thin. This means, the last mentioned set, written as

$$
\left\{k_{1}^{(l)}, k_{2}^{(l)}, \ldots\right\}
$$

is non-thin where $Z=\left(Z_{i}\right)$ is the single sequence refereed to in Definition 1. Therefore there exists $\delta_{l}>0$ such that

$$
\frac{1}{p}\left|\left\{i \in \mathbb{N}: k_{i}^{(l)} \leq p\right\}\right|>\delta_{l}
$$

for infinitely many positive integers $p$. Notice that each $k_{n}^{(l)}$ corresponds to an element $(i j) \in \mathbb{N} \times \mathbb{N}$ so that, to the sequence $\left\{k_{1}^{(l)}, k_{2}^{(l)}, \ldots\right\}$ corresponds a sequence of elements $\left\{\left(i_{1}, j_{1}\right),\left(i_{2}, j_{2}\right), \ldots\right\} \in \mathbb{N} \times \mathbb{N}$.

By the Strong Law of large Numbers

$$
P\left\{u \in U: \lim _{h \rightarrow \infty} \frac{1}{h} \sum_{m=1}^{h} u_{i_{m} j_{m}}=\frac{1}{2}\right\}=1 .
$$

Therefore, by (ii) and (iii) we have: 
If $u \in \mathcal{N}$, then

$$
\frac{1}{P}\left|\left\{i \leq P:(Z(x(u)))_{i} \leq \frac{1}{j}\right\}\right|>\frac{\delta_{l}}{4}
$$

for infinitely many $P$, where

$$
\mathcal{N}=\left\{u \in U: \frac{1}{h} \lim _{h \rightarrow \infty} \sum_{m=1}^{h} u_{i_{m} j_{m}}=\frac{1}{2}\right\}
$$

which prove (1).

We now prove

$$
P\left(\left\{u \in U: \Gamma_{x(u)} \subseteq \Gamma_{x}\right\}\right)=1 .
$$

We call $u=\left(u_{i j}\right)$ "normal", if the single sequence $Z(u)$ is normal, i.e. if

$$
\lim _{n \rightarrow \infty} \frac{1}{n} \sum_{i=1}^{n}(Z(u))_{i}=\frac{1}{2}
$$

where $Z(u)=\left\{u_{11}, u_{21}, u_{22}, u_{12}, u_{13}, \ldots\right\}$, that is $Z(u)$ is formed using our diagram.

$P\left(\mathcal{N}^{\prime}\right)=1$, where $\mathcal{N}^{\prime}=\{u \in U: u$ "normal" $\}$ by the Law of Large Numbers.

Now suppose $u \in \mathcal{N}^{\prime}$ and $L \in \Gamma_{x(u)^{\prime}}$ then for every $\varepsilon>0$,

$$
\left\{i \in \mathbb{N}:\left|(Z(x(u)))_{i}-L\right|<\varepsilon\right\}
$$

is non-thin or there exists $\delta_{\varepsilon}>0$ such that

$$
\frac{1}{P}\left|\left\{i \in P:\left|(Z(x(u)))_{i}-L\right|<\varepsilon\right\}\right|>\delta_{\varepsilon}
$$

for infinitely many. This implies

$$
\frac{1}{P}\left|\left\{i \leq P:\left|(x(u))_{i}-L\right|<\varepsilon\right\}\right|>\frac{\delta_{\varepsilon}}{2}
$$

for infinitely many $P$, or $L \in \Gamma_{x}$. Therefore $\Gamma_{x(u)} \subseteq \Gamma_{x}$ for all $u \in \mathcal{N}^{\prime}$.

Hence (2) is true, completing the proof.

\section{Final Remarks}

As we mentioned, subsequences and statistical cluster points of double sequences could be defined in ways different from those presented here. One could question if our result holds in this different setting.

Further, "almost all" results like the theorem presented here and in [5] could be further pursued.

Finally, the normal numbers in $(0,1]$ have Lebesque measure 1 but are of the first Baire Category. Permutations of sequence could be considered. 
There is a natural way to consider a metric $d$ on $\mathcal{P}$, the set of all permutations of $\mathbb{N}$ in such a way that $(\mathcal{P}, d)$ is a complete metric space (see [4]).

Question: What can we say about the relationship between $\Gamma_{x}$ and $\Gamma_{x(P)}$ ? Here $x(P)$ is the sequence $\left\{x_{P(n)}\right\}_{n=1}^{\infty}$. In light of the proof that the normal number in $(0,1]$ is a first category set, one might suspect that a "permutation analogue" of the theorem presented here is not true.

\title{
REFERENCES
}

[1] H. Fast, Sur la convergence statistique, Colloq. Math., 2 (1951), 241-244.

[2] J. A. Fridy, Statistical limit points, Proc. Amer Math. Soc., 118 (4) (1993), 1187-1192.

[3] K. Krickeberg, Probability Theory, Addison-WesleyPublishing Co. Inc., 1965.

[4] H. I. Miller, Summanbility of subsequences, rearrangements and strechings, Akad. Nauka umjet. Bosne i Hercegovine, Rad. Odjelj. Prir. Mat. Nauka, LXVI, 19 (1980), 95-102.

[5] H. I. Miller, A measure theoretical subsequence characterization of statistical convergence, Trans. Amer. Math. Soc., 347 (5) (1995), 241-244.

[6] H. I. Miller and L. Miller-Van Wieren, Some cluster point theorem, Hacet. J. Math. Stat., 44 (6) (2015), 1405-1409.

[7] H. I. Miller, L. Miller Van Wieren, T. Taş and T. Yurdakadim, Inclusion results on statistical cluster points, Anal. Math. (Szeged), accepted for publication.

[8] H. I. Miller and L. Miller-Van Wieren, Statistical cluster point sets for almost all subsequences are equal, Hacet. J. Math. Stat., accepted for publication.

(Received: July 1, 2016)

\author{
Harry I. Miller \\ A. Šahinagića 8 \\ 71000 Sarajevo \\ Bosnia and Herzegovina \\ himiller@hotmail.com
}

